\title{
14. A TAXONOMY OF DATA COMMUNICATION PROTOCOLS FOR VEHICULAR AD HOC NETWORKS
}

\author{
Yousef-Awwad Daraghmi ${ }^{1}$, Ivan Stojmenovic ${ }^{2}$, Chih-Wei $\mathrm{Yi}^{1}$ \\ ${ }^{1}$ Department of Computer Science, National Chiao Tung University, Hsinchu City 30010, \\ Taiwan, yousef.cs98g@nctu.edu.tw,yi@cs.nctu.edu.tw \\ ${ }^{2}$ SEECS, University of Ottawa, Canada, stojmenovic@gmail.com
}

\begin{abstract}
Vehicular Ad Hoc Networks (VANETs) are the potential core of the Intelligent Transportation System (ITS) which aims to increase people safety and improve transportation efficiency. In this paper, we provide the first known taxonomy of VANET data communication protocols, based on road dimension, neighbor knowledge, acknowledgment, start of forwarding, competition to retransmit, vehicle connectivity, urgency, and message contents. The taxonomy provides fundamental blocks in VANET data communication protocols and help researchers better understand the details of each protocol. Further, the taxonomy helps in designing new protocols, by replacing one option by another from the same category. It also helps to understand various limitations of certain protocols, and the need to generalize these protocols and apply them in various VANET applications and scenarios. We also provide here a survey of recent data communication protocols, and a tutorial on how most of them work.
\end{abstract}

\subsection{Introduction}

Vehicular Ad-Hoc Networks (VANETs) are distributed, self-organized and potentially highly mobile networks of vehicles communicating via wireless media. VANETs are a form of Mobile Ad hoc Network (MANET) where movement of each node (vehicle) is restricted by road direction, encompassing traffic and traffic regulations. These restrictions introduce new challenges to MANET described as high dynamicity of the underlying topology and intermittent connectivity [RRSS]. Movement direction of vehicles is predictable to some extent and power consumption can be compensated by their batteries $[\mathrm{BF}]$.

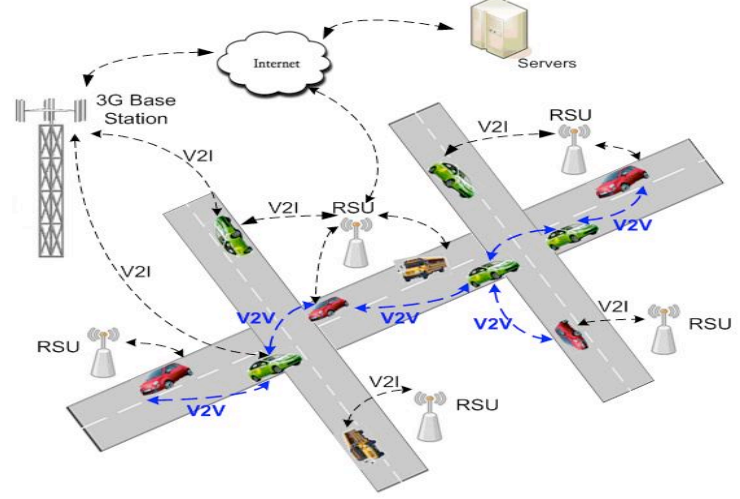

Fig.1: ITS application system model

Industry and academia have invested in VANETs since they are a major part of Intelligent Transportation Systems (ITS). The ultimate ITS goals are to improve safety on roads and provide better services and traffic management. The ITS is an integrated flexible and scalable architecture, illustrated in Figure 1. In this system, infrastructure-based protocols such as 
cellular communication protocols, and vehicular ad hoc network (VANET) protocols are being integrated to facilitate information collection process. Road-side units (RSUs) provide direct wireless communication from nearby vehicles to the infrastructure (V2I). They are currently very sparsely deployed. Their ubiquitous deployment is futuristic and expensive. Vehicle to vehicle $(\mathrm{V} 2 \mathrm{~V})$ communication is applied when a vehicle is not directly connected to an RSU. Hybrid communication protocols rely on both inter-vehicle (V2V) and road-side (V2I) access. This scenario assumes that each vehicle is equipped with a central processing unit to run protocols, a wireless transceiver, a GPS receiver (to provide information on location), sensors to measure various parameters, and an input/output interface for human-vehicle interaction, with small additional hardware cost for car manufacturers.

V2V and V2I have different functionalities and advantages. V2V offers advantages in supporting time-critical safety applications (collision avoidance), broadcasting abrupt events, informing of nearby business activity (stores can deliver ads from their RSUs), provisioning related localized service, and avoiding infrastructure cost and user fees. The advantage of V2I over $\mathrm{V} 2 \mathrm{~V}$ is the provision of Internet applications, global traffic coordination and prediction (by data fusion of information collected at control centers), reliability, technical simplicity, QoS guarantees, wider coverage and professional maintenance.

Our main goal is to describe protocols for delivering timely information (e.g. about traffic congestion, weather, road conditions, and location based services) to drivers in a cost-effective manner. For example, V2V communication is used to inform vehicles approaching a congested area, so that they can adjust their itineraries. These protocols are envisioned as basic ingredients in a number of additional applications, such as finding a parking lot, restaurant, gas station, or receiving traffic flow advice, crash warnings, cooperative adaptive cruise control, speed limit warnings, animal warnings, rescue and authorized vehicles assistance, fleet-social networking, map and media updates, mobile commerce, certificate updating etc. Applications need a minimum share of vehicles equipped for $\mathrm{V} 2 \mathrm{~V}$ communications. Diffusion is an example of single-hop V2V, where each vehicle transmits content beacons periodically. In proposed DSRC medium access standard (discussed bellow), each vehicle sends periodic messages containing vehicle's position, velocity, direction, etc. These mandatory beacons can be used in diffusion. An application collects data from neighboring vehicles, aggregates and stores data into tables, and then transmits current tables to neighbors, updating their tables.

Applications related to VANET are generally classified as traffic safety and non-safety applications. Safety applications attempt to inform drivers of safety messages urgently (minimum time delay) $[\mathrm{BM}][\mathrm{CNS}]$. They are based on monitoring vehicle and road conditions, and other vehicles. In case of emergency, vehicles exchange messages and co-operate to help each other. Examples of traffic safety applications are cooperative collision avoidance, pre- or post-crash warning and rollover warning. When a vehicle detects an abnormal condition, an event-driven message is generated and disseminated through relevant portion of the vehicular network with the highest priority [DDB][BTD][YMF].

Non-safety applications provide drivers and passengers with information related to traffic efficiency and entertainment. They are also called comfort services [YMF] and general information services [WTM], and they include traffic efficiency applications such as better route (road) selection, better traffic balance and shorter travel time [CNS]. The focus of non-safety applications is not only delivering information to the largest number of vehicles over large areas [WER][NSI][CGM], but also applications that target small areas to enable cooperative driving 
[TAF]. It also includes value-added applications such as entertainment and business advertisements [CNS][WTM].

In this paper we provide a novel (and first, to the best of our knowledge) taxonomy of VANET data communication protocols. We examine several types of multi-hop communications (e.g. one-to-one, one-to-some, and one-to-all), required by novel ITS, and explain the ingredients of the most relevant existing communication protocols. We outline existing solutions and highlight their drawbacks.

\subsection{Taxonomy of VANET communication protocols}

VANET applications require communication among vehicles to enable data dissemination. Different traffic scenarios require different communication characteristics. Based on VANET characteristics and conditions, we provide the following taxonomy for VANET data communication protocols.

\subsubsection{Defining and naming problems}

We consider the two main types of multi-hop communications, routing (one-to-one) and geocasting (one-to-all in a specific region), and their combination for a content dissemination application (one-to-some). Unicast routing is used for applications that require one-way or twoway communication from a vehicle to/from a RSU, or communication between two remote vehicles, for coordinating response teams, tracking a car, or allowing two drivers to communicate. Routing applications also include delivering ITS services to end users, facilitating service providers to post their services, supporting Internet connections between users and service providers. The routing task is challenging because of high mobility and intermittently connected vehicular networks. The task can be specified according to assumptions made and the application context. The destination of a message could be a fixed geographic location, such as an access point (RSU), or a moving car whose location is known and is being updated based on reported speed and direction. A moving destination may also have unknown location, i.e., police searching for a car. Vehicles could move with their plans of movement being sent to a centralized location and/or being available to nearby vehicles, or without reporting their plan of movement. The source and destinations may or may not be always connected via other cars.

RSUs and/or neighboring vehicles can be used to facilitate the routing process. If RSUs are connected and can communicate fast among themselves, then they can assist routing as proposed in [WK]. Gateways (RSUs) provide shortcuts to routing. Vehicles route toward the nearest RSU (which is routing task itself), and the message is delivered to destination from its nearest RSU. In the rest of this paper, RSUs are not used in routing process. Routing algorithms are then generally resolving three issues: finding the destination (FD) (if unknown), small scale routing (SS) (between two road intersections), and large scale routing (LS; decisions made at road intersections). Applications like parking slot reservation require two-way routing and integration of the Internet Protocol (IP) stack with routing protocols.

The second problem is how to spread a message from a source vehicle to all other vehicles. It has different names in literature: broadcasting, flooding, data dissemination, warning delivery, and geocasting. Broadcasting spreads the information without considering the region borders. Flooding refers to the single retransmission of a message by all recipients. Geocasting (G) is (arguably) a geographically-constrained form of limited broadcasting. It involves the dissemination (delivery) of information to all vehicles on a road segment or in a given geographic area, the suppressing of multiple repetitive warnings for the same event, and the 
determining of boundaries for spreading warnings. Geocasting is initiated by a vehicle or a RSU. The source of information intended for geocasting may or may not be located in the geocasting region. For example, reports on congestion on a highway segment may be useful to vehicles approaching it, and not necessarily to the vehicles already in the congested area. Such 'remote' geocasting algorithm may consist of a routing task to reach the area of interest before flooding it, followed by limited broadcasting inside that area. Another example is content dissemination, which is based on file dissemination, with the file being divided into multiple messages [LCM]. The opportunistic content information (e.g. about a gas station) needs to be preserved in a persistence area of approaching vehicles.

\subsubsection{Road dimension}

There are different scenarios in which a vehicle can move on roads. In the basic scenario, vehicles move along a highway with no intersections. Such two-directional road without intersections is considered one dimensional (1D). A one-directional road segment between two intersections is also 1D. More general scenario places cars in two dimensions (2D) in urban areas where there are many intersections and a vehicle can change the road. There are also some intermediate scenarios where vehicles are restricted by road junctions or roundabouts. An ideal protocol should be able to automatically adjust its functionality to a given road dimensionality. Some protocols address only a 1D scenario and are incomplete when considered in 2D. Protocols addressing 2D case are more general, and are also applicable when the actual scenario is 1D.

\subsubsection{Neighbor knowledge}

In some VANET communication protocols, vehicles make forwarding decisions without neighbor knowledge (NN). In others, it is necessary for a vehicle to learn about its neighbors. The vehicle may know the position, velocity or other parameters of neighboring vehicles. Such neighbor positional knowledge (NP) becomes available when all vehicles exchange information about each other by periodic beacons. Communication among vehicles in VANETs may be performed by means of the Dedicated Short-Range Communication (DSRC) standard that employs the IEEE 802.11p for wireless communication. DSRC is a Medium Access Control (MAC) protocol that operates at $5.9 \mathrm{GHz}$.

\subsubsection{Acknowledgments}

Acknowledgments are used to inform a transmitting vehicle that the transmitted message has been received. Some protocols do not use any acknowledgments in decision making process $(N A)$. We use the term beacon acknowledgment $(B A)$ when a node explicitly acknowledges the reception of a message by adding the message receipt to its regular beacons. Inter beacon interval could be of a fixed or an adjustable length. Message receipts could be sent also independently of beacons. Forwarding nodes normally assume that neighbors received the message until their next beacon is received, or a timeout expires. The status of a transmitted message may be predicted by other means. A vehicle may estimate the reception of a message based on the distance from the sender, or the vehicle waits to hear the same message transmitted by others (passive acknowledgment). In our classification, the term $P A$ refers to the implicit (passive) acknowledgments, while the term $R E$ (reception estimation) refers to reception estimation based on the distance from the sender to that neighbor. 


\subsubsection{Starting forwarder selection}

Vehicles are normally in idle state with respect to a message being broadcasted. In this state, either there is no message copy received (so retransmission is impossible), or there is no need to retransmit (e.g. when all known neighbors acknowledged the message reception). Otherwise, the question is how a vehicle recognizes the need for message retransmission (either by itself or by a neighbor), to cover certain vehicles. This represents recognition to start the process of selecting forwarding neighbors, and an active state with respect to the message.

When does forwarder selection process start, that is, when a vehicle moves from an idle to an active state? We now describe several options that are not necessarily mutually exclusive (that is, the same protocol may apply few of them). The simplest decision is that a vehicle enters an active state each time the message is received (this is refereed to as $A A$ - always activate) [FM]. Other option is to immediately activate (IA) after receiving the message for the first time and ignore any event or further receptions of the same message [SYK]. It is applied in flooding protocol, where each node will retransmit the message exactly ones. Another option is that a forwarder enters an active state whenever another node (e.g. new neighbor) sends a beacon with the missed message ID [RRS]. We refer to this scheme as lack of acknowledgment (LA). Therefore, a vehicle may restart the process after an idle period (believing that all neighbors are aware of the message) if a new neighbor emerges. Finally, a vehicle may become active if it receives a message from a neighbor which does not cover all its neighbors [AOS]. Node $A$ is believed to cover node $B$ if the distance between them is less than a threshold value. This method is referred to as $N E$ (neighbor elimination), and is based on applying $R E$ or $B A$. Note that the recognition of a new neighbor or the reception of the message for the first time does not always move a node to the active state in this $N E$ scheme. The emergence of new neighbors (in need of message, based on local information) may also activate the node.

\subsubsection{Compete to retransmit}

An active vehicle is a candidate to retransmit, but is not in the process of retransmitting yet. If retransmission is only subject to MAC layer details then the protocol is classified as $N C$ (no competition). Normally vehicles 'negotiate' the task of retransmitting with neighbors. Such negotiation is often carried by starting timers (not necessarily simultaneously), and waiting for the timers to expire. Active nodes ('winners' of forwarder selection processes) will enter the transmission state following a MAC layer algorithm, since an immediate retransmission by two or more nodes (following their simultaneous reception) will cause collisions, as VANET communication is envisioned on a single channel. However, medium access protocols introduce certain delays that allow nodes to respond at different times. After one of them starts retransmitting, others wait for the completion of this transmission before continuing. Sometimes, the retransmission may become even obsolete (causing node to cancel its retransmission and enter idle state). Strictly speaking, once a node passes a message to the MAC layer, there is no cancellation of the packet until there is some limit on how many times it can be retransmitted (after a failure). Any cancellation based on overhearing other data is done at the network layer. However the desired cancellation at MAC layer can be achieved by a suitable cross layer design.

Several MAC layer protocols were applied in literature. The proposed DSRC standard is based on the 802.11 type of competition. Nodes will wait for certain number of collision free slots before their own retransmission. The waiting time is decided by some randomness or a priority based rule. Since a time delay function (expressed in number of slots) is discretized, it may be possible that two cars select the same waiting time and therefore their retransmissions 
collide. This basic scheme does not offer recovery from this collision, and is normally applied to the broadcasting task where a single transmission normally triggers multiple retransmissions. An alternative (more convenient for routing task where single transmission by a node is followed by single retransmission of the selected forwarder) is to apply Ready-to-Send Clear-to-Send (RTSCTS) protocol. RTS and CTS signals are of fixed length. In case of collisions of CTS responses, competition repeats with new random backoffs (possibly exponentially increasing in length) until selection of forwarder is unique and confirmation from the sender node is received. Sender node then transmits full message with forwarder node being selected. We denote these schemes $T D$ (time delay), which can be based on DSRC (TD-D) or RTS-CTS $(T D-R)$.

Another MAC scheme frequently applied in VANET communication protocols is based on black burst $(B B)$ [SWSG]. Active node will transmit a noise signal for certain variable time duration. It will start transmitting if, after stopping, it hears no noise from other vehicles.

\subsubsection{Connectivity}

Connectivity is an important metric in VANETs since vehicles tend to be disconnected even in dense traffic [AOS]. However, some communication protocols assume that vehicles are connected all the time. Under this assumption, the performance of a communication protocol may drop if a disconnection occurs. Therefore, other communication protocols propose solutions for vehicle disconnection by providing alternative schemes such as the store-carry-forward scheme. When vehicle disconnection is handled by a protocol, we refer to it as intermittent connectivity (IC) scenario. We use the term always connected $(A C)$ when vehicles are assumed to be connected all the time in a given protocol.

\subsubsection{Urgency}

An important feature of safety applications is urgency in which an extent message should be delivered from a source to a destination with minimum time delay. Safety applications (e.g. crash warning) require message delivery in the shortest time, while non-safety applications require reliability to achieve the highest percentage of message delivery. For example, an accident report is extremely urgent to be forwarded to incoming vehicles toward the accident, but it is much less (but still) urgent to quickly forward the accident position to emergency centers. Although timely distribution remains always a goal, most applications allow for some time window for their reception, and the main goal of the protocol is then to provide high reliability. For example, an advertisement has to reach all vehicles in a specific area. We use the term time critical (TC) when a protocol aims to achieve fast message transmission and we use the term reliability oriented $(R O)$ when a protocol aims to primarily guarantee the reception of each vehicle.

\subsubsection{Message contents}

There are different message types addressed in VANET communication protocols. We classify these types into two categories. The first category is full message $(F M)$ which is the original message, possibly containing also fixed amount of information about the sender and receiver, such as sender position, destination position, and velocity [OLL]. In the second, a message may include the ID of the dedicated forwarder(s) to ensure quick and reliable message delivery $[\mathrm{SYK}]$. We refer to this category as forwarder attached $(F A)$. Longer messages have smaller packet reception rates, therefore impacting the performance. 
Forwarder attached (FA) based algorithms are alternatively called sender-oriented. Sender node decides which of its receivers will forward the message. Full message (FM) based algorithms are alternatively classified as receiver-oriented. Sender-oriented approaches are generally less reliable, because the sender does not have accurate information about the nodes in need of message, which are neighbors of receivers. For instance, if sender $S$ has two neighbors $A$ and $B$, whether or not further retransmissions are needed, and by which of neighbors, depends on their own neighborhood. If $A$ has neighbors $C$ and $D$, while $\mathrm{B}$ has neighbor only $D$ then retransmission by A is more beneficial. However $S$ is not aware of $C$ and $D$ and therefore is unable to make best decision on retransmission by $A$ or $B$. This point was well elaborated and illustrated in [LYS].

The above nine categories, in our taxonomy, outline a detailed description of VANET data communication protocol functionalities, operations, and major characteristics. In the remainder of this article, we review several VANET data communication protocols and highlight the taxonomy. We outline the application domain targeted by each protocol and its characteristics.

\subsection{Reliability oriented geocasting protocols}

We divide existing geocasting into two groups, depending on urgency metrics. This section discusses reliability oriented (RO) protocols, while the next section discusses time critical (TC) solutions.

Most existing reliability oriented solutions (c.f. [CNS]) do not resolve temporary disconnection from the source node. These protocols also do not make use of RSUs, and assume that all vehicles belong to the same connected cluster. Once a message reaches the back of a cluster, forwarding is stopped. For example, [FM] designed and analyzed a simple warning delivery service protocol. In the proposed protocol, every time a vehicle in the safety area receives a new warning message, it decides, with probability $p$, to act as relay and forwards the message. Vehicles outside the safety area, even if informed, do not relay the warning message. There are few broadcasting cycles, which start at regular intervals every $D$ seconds. The question of which a vehicle initiates a broadcast cycle remains unresolved in [FM]. The protocol may unnecessarily send too many messages after all vehicles already have the message. Also in other scenarios it may send too few messages, because there is no mechanism to restart flooding immediately upon the discovery of new neighbors.

1D broadcasting algorithm in [LLZ] disseminates the same message to all cars on a road segment. As in [SFLYOF], the furthest node from the sender retransmits the message for fast progress. The extension is that the node closest to the middle between two senders retransmits for increasing reliability.

\subsubsection{Reliable and efficient broadcasting in VANETs (ackPBSM)}

Parameter-less reliable broadcasting strategy with acknowledgment (ackPBSM) targets different (e.g. 1D, 2D) traffic scenarios [RRS]. This protocol aims to guarantee message reception and reduce the number of retransmissions. It only employs local information acquired via periodic beacon messages. Beacons include a sender's position and acknowledgements of circulated broadcast messages $(B A)$. Using the former information, each vehicle decides whether it belongs to a connected dominating set (CDS) [SSZ]. A set is said to be dominating if it is connected, and each node either belongs to it or has a neighbor that belongs to it. As a part of the $5.9 \mathrm{GHz}$ DSRC standard, each vehicle emits a hello message (or beacon) periodically (e.g., every $300 \mathrm{~ms}$ ), and afterwards the construction of the CDS has no message overhead, which is the main 
advantage of [SSZ]. CDS is useful in dense networks, such as cars waiting at traffic lights in cities, to avoid collisions by many retransmissions, which will occur even in existing solutions where each car retransmits at most one (since in such scenarios they may not be located on a single lane).

Every node has two lists: $R$ (nodes assumed to have received the message) and $N$ (other 1-hop neighbors). After receiving any copy of the same message, receiving cars discard neighbors covered by the same transmission (based on their estimated location at the time of message transmission). That is, node puts its neighbors that are covered by the source in the $R$ list. Vehicles in the CDS use a shorter waiting period before deciding if they should retransmit such a message. The actual time delay formula may include number of nodes in $N$ (e.g. proportional to $T=1 /($ number of nodes in $N)$ ), distance from previous senders, and other factors. One of the basic ingredients is the neighbor elimination algorithm [SSZ]. At the end of this backoff (defer) time, a car will retransmit the message only if it believes that at least one of its neighboring cars is not covered by any of the received message copies. That is, when the waiting period expires, a vehicle retransmits only if it has one or more neighbors who did not acknowledge message reception within the last circulated beacon ( $N$ is then nonempty). Afterwards, all neighbors are added to the $R$ list and the retransmitting node waits for another time period to receive acknowledgments from neighbors. If a neighbor does not send an acknowledgment, this neighbor will be deleted from $R$ and moved to $N$. Also, as new neighbors appear, the evaluation timer can be restarted. In this solution, the road structure and mobility information are not used, and it is therefore applicable to arbitrary vehicle locations.

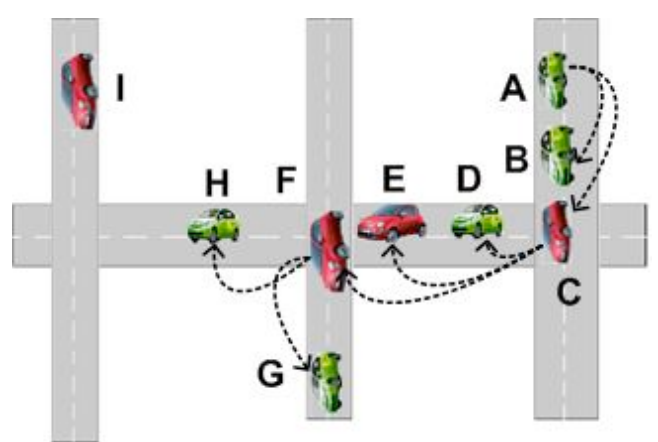

Fig. 2: ackPBSM- reliable broadcasting.

The waiting period is useful to consider new vehicles arriving later. The protocol also makes use of the store-carry-forward concept to overcome the intermittent connectivity problem. Figure 2 shows a 2D example of the ackPBSM protocol. All nodes exchange one-hop information (NP). Nodes $C$ and $F$ and $I$ are in CDS of its connected component. Vehicle $A$ is the source and transmits to $B$ and $C$ (in its range). $B$ assumes that its only neighbor $C$ got the message and holds activation. $C$ is activated since its neighbor $D$ is likely not covered $(N E)$. $C$ retransmits, and $B$ then cancels its retransmission. $C$ retransmits and $B$ then cancels its transmission. Vehicles $D, E$ and $F$ receive the message. $F$ retransmits first because it is in CDS. $D$ and $E$ cancel their transmissions upon receiving message from $F$. Reliability is guaranteed by mandating each receiver to send an acknowledgment. If $B$ drives towards $G, B$ will overtake $C-F$. Although $B$ and $D-G$ are not neighbors, $B$ will not retransmit to $D-G$ if they acknowledged the reception of the message. If one node did not receive the message, its neighbor would detect it because of the lack of acknowledgment $(L A)$ and retransmit the message to that node. If $H$ drives 
towards $I$, since $I$ did not acknowledge the message, $H$ will retransmits the message to $I$. Therefore ackPBSM protocol has the ability to work in an intermittent connectivity scenarios (IC). The main drawback of the protocols is the overhead resulted from acknowledgment piggybacking in beacon messages. The time delay function needs to be better specified for time critical applications.

\subsubsection{Persistence-based protocols}

Three probabilistic and timer based broadcasting suppression techniques for wellconnected vehicular networks have been proposed in [WTPMBS] to minimize the well-known broadcast storm problem. These techniques focus on achieving a high percentage of emergency message delivery and keeping end-to-end delay at acceptable levels in a highway scenario with no intersections. Neighbor knowledge is not required and receptions are not directly acknowledged. Also, collision may occur if two nodes in the same slot decide to retransmit.

In the weighted $p$-persistence scheme, upon receiving a packet, a node $j$ waits for a constant time $W$ to receive other potential copies of the message. Let $i$ be the closest neighbor from which the packet has been received. Then, if $j$ receives the packet for the first time, it rebroadcasts the packet with probability $p_{i j}=D_{i j} / R$ where $D_{i j}$ is the distance between $i$ and $j$, and $R$ is the transmission radius. $j$ discards duplicated packets. If $j$ decides not to retransmit, it waits for an additional time $\delta$, which accounts for transmission and propagation delays, to overhear the same message again from any neighbor. If this is not the case, $j$ rebroadcasts with probability 1. In the slotted 1-persistence scheme, $j$ selects time slot $S_{i j}=N s(1-D i j / R)$, where $N s$ is the maximum number of slots. It rebroadcasts (with probability 1 ) at the assigned slot if it receives the packet for the first time and does not hear any duplicate before the assigned slot; otherwise the packet is discarded. Finally, in the slotted p-persistence scheme, rebroadcasting is done with pre-determined probability $p$ instead of probability 1 , and retransmission with probability 1 is scheduled if no duplicate was heard within certain time limit. Versions of the algorithms using the Received Signal Strength (RSS), instead of position information, were also described.

\subsection{Time-critical geocasting protocols}

Basic algorithm in [BTD] focuses on cooperative collision avoidance (CCA). When a car detects an accident, it starts to forward a "wireless collision warning message" at regular intervals, with the goal of avoiding chain collisions. A naïve blind flooding algorithm is discussed, where each vehicle that receives a warning message starts decelerating and forwarding the message if it comes from its front. Algorithm has similar characteristic as probabilistic algorithm [FM] that we discussed already.

\subsubsection{Multi-hop vehicular broadcast (MHVB)}

$M H V B$ algorithm [OLL] aims to periodically forward safety-related messages within an allowable time delay (TD). Nodes further than threshold distance do not compete to retransmit the messages (despite possibly receiving it). Receivers calculate the waiting time based on the distance to the source, so that further nodes wait less time. If a node receives two or more copies of the same message, it checks if it is inside a circle with any two senders as the diameter, and it cancels retransmission if so. Otherwise it retransmits after the waiting period expires. We notice that neighbor knowledge is not required. A node only needs to know a sender's position which is attached with the message.

$M H V B$ decides that a vehicle is in a congested area if vehicles have slow speed, the 
number of neighbors exceeds a threshold value, or the number of vehicles in front of and behind the vehicle exceeds a threshold value. Congestion is generally detected by short range sensors. The waiting time of congested vehicles is expanded, and it is inversely proportional to the number of vehicles around. This increases the time delay and contradicts the MHVB original motivation which is retransmitting effectively sooner not later.

\subsubsection{Emergency message dissemination with ACK-overhearing based retransmission (EMDOR)}

After an originator broadcasts an emergency message, in the EMDOR algorithm [SYK], a relay node immediately retransmits it. Relay node can be selected using any existing method. In particular, $[\mathrm{SYK}]$ uses the $p$-persistence method [WTPMBS]. In that method, a forwarding area is divided into slots, and nodes apply a probability $p$ to relay, starting from the furthest slot, with a certain inter-slot delay before nodes in the next slot consider transmitting. Note that there could be collisions among few nodes in the same slot if both decide to retransmit, or failure to transmit when some nodes indeed exist in a slot. After retransmitting, the relay node sends an ACK to reply to the originator. It serves for other nodes to learn about the message if they receive ACK but not the message. In that case they can send a request to the relay node to retransmit again.

$E M D O R$ suffers from collisions between two nodes in the same slot if both decide to retransmit. A possible failure is when a node does not receive a message and also the acknowledgment related to that message. Although this protocol achieves higher reliability than other protocols, it suffers from high overhead because every message is encapsulated with additional information such as a message originator's address, a broadcast identifier and a relay node location.

\subsubsection{Distributed fair power adjustment protocol (D-FPAV)}

[TMSH] first discusses the distributed fair power adjustment algorithm (D-FPAV) for sending beacons. Using information gathered from beacons, each node applies the 'water filling' approach, increasing its power as long as the minimal beaconing load condition is satisfied. These power levels are then exchanged with neighbors. The node then selects the minimal minimum power level among the locally computed and those by the surrounding vehicles. Then, [TMSH] discusses the emergency dissemination for vehicular environments algorithm (EMDV). A sender node reduces the communication range and allows only neighbors within the smaller forwarding range to retransmit. The sender pre-selects its next hop, as the furthest vehicle within its forwarding area. Each node within the forwarding area after receiving the message, will count the number of received or sent copies of the same message. The dedicated forwarder retransmits immediately (if it has received a message). All receivers enter contention with time delay proportional to 1-progress/ForwardingRange; that is, according to their distance from the sender. The contention will restart after each node has received a copy of the same message, according to the distance from the latest sender. There are no acknowledgments.

Simulation results show that the best performance can be achieved when D-FPAV and $E M D V$ are combined because $D-F P A V$ ensures that the channel busy time is kept to a level on which $E M D V$ can operate efficiently. A problem in this protocol is large message overhead caused by the large size of beacons or the need for sending additional beacons. Also, this method may unnecessary cause failures of alert messages when the forwarding area is empty and there are still vehicles within the communication range of the sender. 


\subsubsection{Receiver Consensus (ReC)}

[LYS] proposed Receiver Consensus ( $\mathrm{ReC})$ algorithm, which exploits geographical information to help nodes autonomously achieve agreement on forwarding strategies. Each forwarding candidate ranks itself and its neighbors (who affirmatively or potentially received the message already) by distance to the centroid of neighbors in need of message, to assign different priority in forwarding among neighboring nodes and remarkably suppress unnecessary retransmission, while enabling best nodes to transmit the packet without waiting. The effectiveness and efficiency of this method is validated through extensive simulations under $802.11 \mathrm{p}$ settings. The results demonstrate that the proposed protocol achieves the high reliability of leading state-of-the-art solutions, while at the same time significantly enhances timeliness, dedicating itself to disseminating emergency messages in $2 \mathrm{D}$ vehicular networks.

\subsection{Small scale routing protocols}

Recall that small scale routing deals with routing between two road intersections, with vehicles being in several lanes and possibly in opposite directions. In this section we review existing small scale routing protocols.

\subsubsection{DPP and OPERA}

The Directional Propagation Protocol (DPP) scheme which is dedicated to multi-lane highway scenarios is proposed in [LA]. It assumes disconnected clusters of vehicles. Oncoming clusters are used as bridges to inter-connect two successive co-directional clusters of cars driving toward a destination intersection. They act only as bridges between two co-directional clusters, without taking custody of the message. This idea was subsequently used also in the opportunistic packet relaying algorithm (OPERA) for temporarily disconnected vehicular ad hoc networks [AOS]. OPERA is a small scale routing where source and destination are on the same path between two intersections. Beacons are used to construct and maintain clusters where vehicles receiving no beacons ahead of them become cluster heads. It was proven that disconnection is likely even in relatively dense traffic [AOS].

The baseline step of OPERA consists of message forwarding from a source to its neighbors within the cluster. A cluster head carries the message. Advance is possible as long as the cluster is connected with other clusters on the same path. If not, clusters in the opposite direction are used. A message is transmitted by any node that has a neighbor in other clusters. It is forwarded among nodes in the cluster until it reaches the destination. If clusters cannot advance the message towards the destination, the message is returned to the cluster head for data muling.

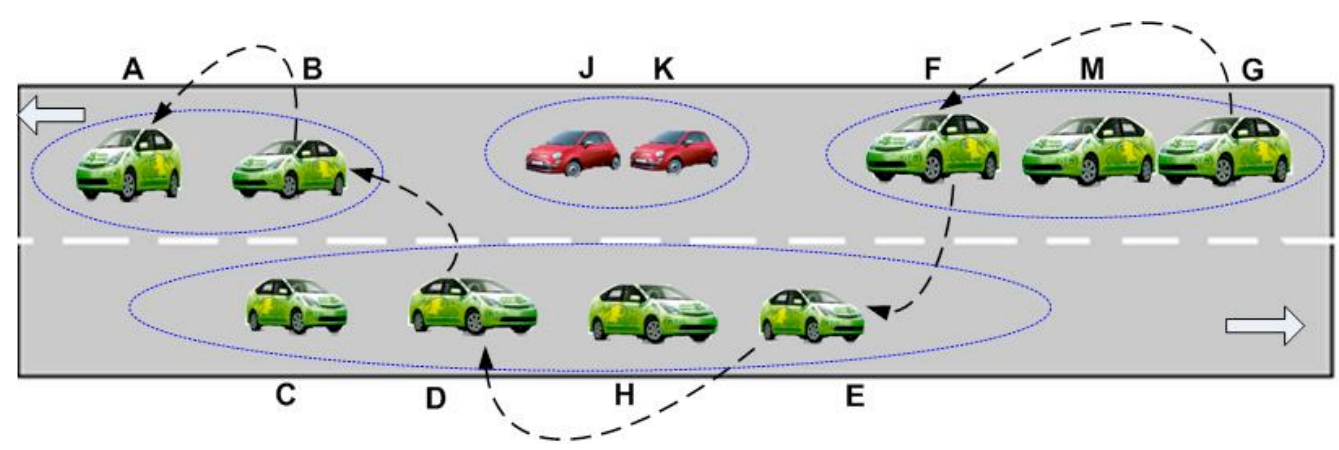

Fig. 3: OPERA- clustering and advancing in small scale routing with the assistance of vehicles from the opposite direction 
Four clusters are shown in Figure 3. Cluster is a chain of co-directional vehicles so that any two consecutive ones are within communication range. Vehicle $A$ is head of a cluster (containing also only car $B$ ) since $A$ does not receive any beacon from a co-directional car in front of it. For the same reason, $E, F$ and $J$ are heads of three other clusters. Clusters headed by $F, J$ and $A$ overlap with cluster headed by $E$ which moves in the opposite direction. Let's assume that $G$ forwards a message to a destination ahead. The message is first forwarded (in one or more hops) toward its clusterhead $F$. $F$ will then store and carry message until there is an opportunity to advance the message. If a node on the route from $G$ to $F$ has a neighbor from a cluster headed in the opposite direction then an advance will be attempted. In this example, after carrying the message for some time, $F$ meets another clusterhead $E$. Message is then transmitted from $E$ to node $D$ in the same cluster which has a neighbor $B$ in the cluster moving in the desired direction. From $B$ message is forwarded toward the clusterhead $A$. However if cluster headed by $E$ does not overlap with any advancing cluster then the message is carried and returned back to cluster headed by $F$ before their disconnection (e.g. from $C$ to $G$ ). Note that in this example cluster headed by $J$ was not used; this is possible if cars within cluster $E$ exchange information about all neighboring clusters from the opposite direction as part of their periodic beaconing.

OPERA does not describe MAC layer details e.g. how to compete to retransmit. The algorithm requires some information exchange about neighboring cluster structure, to decide the proper cluster for advancing, e.g. containing destination or providing the largest advance. Beacons can get lost due to the probabilistic nature of reception, which causes vehicles to have inaccurate estimation of existing neighbors and their positions. Increasing beacon frequency does not resolve the issue [CRR]. Velocity vectors should be piggybacked to beacons, to allow vehicles to estimate positions at the exact time they are needed. Beacon-less receiver based next hop selection strategy is advocated in [CRR].

\subsubsection{Binary partition assisted broadcast (BPAB)}

$B P A B$ (Binary Partition Assisted Broadcasting) [SWSG] is proposed to reduce the delay time for emergency messages in one dimension scenario. Neighbor knowledge is not required in $B P A B$. The protocol is based on iterative binary partition to find the furthest segment containing a possible forwarder. $B P A B$ applies binary partition of the set $I$ initially containing vehicles located between sender $S$ and furthest advance $S+R$, where $R$ is the transmission range. Repeatedly, $I$ is divided into two spatially equal halves (by mid range distance) $I=C+F$, containing vehicles closer and further to $S$. Vehicles from $\mathrm{F}$ compete by black burst transmissions. If there was a collision then $I=F$. In case of silence then $I=C$. If there was a unique black burst then the forwarder vehicle is identified. If the number of iterations reaches a parameter value $N$, then iterations terminate, and the remaining competing cars apply random backoffs in a contention window. $B P A B$ did not address intermittent connectivity directly. However, a car that receives no response to its forwarding request may carry the message and retry later. 


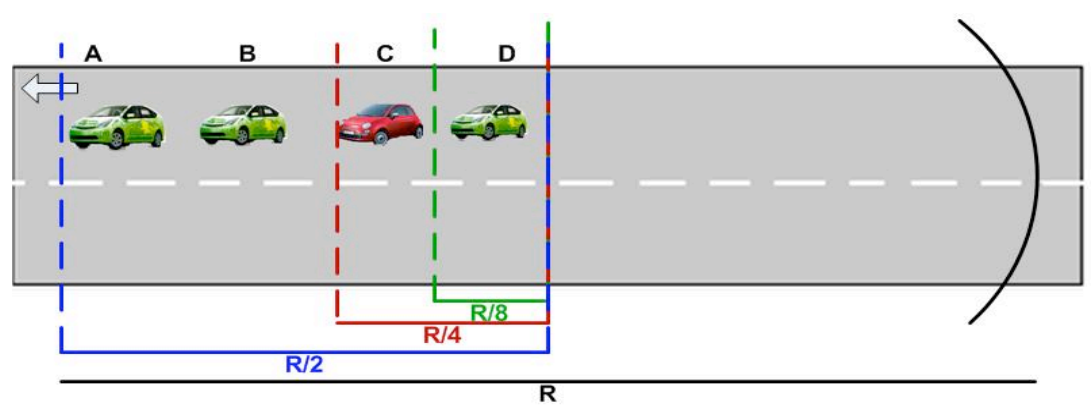

Fig. 4: $B P A B$ - small scale routing

An example of $B P A B$ is shown in figure 4. Initially, the coverage range $R$ is divided in two halves (inner and outer). Black burst (BB) is used to determine which half is the input for the next iteration. All vehicles in the outer half transmit a short fixed length message. At the same time, vehicles in the inner half listen. If there is black burst, vehicles in the inner half will exit the competition and the outer vehicles will become the input for the next iteration. If there is no black burst (outer half is empty), the inner vehicles will become the input of the next iteration. According to $B P A B$, after $A$ transmits RTS, the transmission range of $A$ is divided in the first iteration into two equal segments $(R / 2)$. In this example, the outer $R / 2$ segment does not contain any vehicle, so the inner segment will become the input for the next iteration. In the next iteration, $R / 2$ segment is divided into two equal segments $(R / 4)$. In both $R / 4$ segments, there are vehicles $(B, C, D)$. Since the vehicle in the inner segment $(B)$ detects black burst transmitted by the outer segment, $B$ exits the competition and the outer segment becomes the input for the next iteration. Again, $R / 4$ is divided into two segments equal to $R / 8$. Both $R / 8$ segments contain vehicles $(C$ and $D)$, so black burst is used again and the vehicle $D$ wins to be the forwarder because it is in the outer $R / 8$ segment. Vehicle $D$ sends CTS to $A$ which broadcasts the message attaching the address of the next forwarder $(D)$. $A$ waits for a time period to receive CTS and if not, $A$ resends RTS again. Passive acknowledgment (PA) is used by allowing $A$ to wait for a time to hear another RTS from the forwarder.

$B P A B$ has several drawbacks. Because of reception failures, the best forwarder may not receive the message in the proper iteration, and is not supposed to interfere later with further progress. If all possible forwarders are near the sender, there is a delay until iterations eliminate space with no vehicles. When two cars drive in parallel, both will be selected to forward in parallel, leading to collisions (to resolve this problem, in this and some other protocols, a random delay component should be added to their responses [TJCWL]). Finally, there are no acknowledgments, and no attempts to resend a message because some vehicles located between the two latest senders did not get the message. Therefore, although the authors claim to solve broadcasting problem, in reality they address only small scale routing problem.

\subsubsection{Track detection and distance defer transmission protocols (TRADE \& DDT)}

$T R A D E$ and $D D T$ are two routing algorithms specifically designed for vehicular networks and described in [SFLYOF]. Both solutions are designed to function for cars located in one (or few parallel) lanes on a highway (1D), all driving in the same direction. In TRADE which is sender oriented, a car that retransmits the message will include, in the message, the ID of furthest neighbor (in the direction of message broadcasting). That neighbor, upon receiving the message, will be the next to retransmit the message. The assumption is that other cars between the two are not needed for retransmissions. The intended neighbor may be disconnected at the time of 
message transmission, since the connectivity was established earlier. This would stop the flooding process prematurely.

The $D D T$ solution in [SFLYOF] is receiver oriented: a retransmitting car merely appends its own location with the message. Receiving cars defer retransmitting for a back-off time that is inversely proportional to their distance from the retransmitting car. Therefore the farthest receiver would retransmit first.

\subsubsection{Connection based restricted forwarding (CBRF)}

In the $C B R F$ [WXC], an algorithm is proposed to reduce packets congestions and overhead. Only vehicles located at distance larger than $r$ from the sending vehicle can retransmit where $r$ is less than the transmission range $R$. A forwarding node will choose the next hop as the node progressively closest to the destination among non-congested nodes. However this does not help much since any transmission in vicinity of a vehicle also impacts its congestion and ability to receive other messages at the same time. Congested vehicles may not hear retransmission requests and therefore may not compete to retransmit.

\subsubsection{Distributed vehicular broadcast (DV-CAST)}

In routing algorithm [TWB], upon receiving a packet, a vehicle inside a cluster retransmits with a certain probability. A neighbor vehicle in the opposite direction retransmits, and discards message if moving in same direction as the original message source. A vehicle in the same direction retransmits only if it does not hear the message transmitted by a vehicle in the opposite direction. A cluster-head vehicle and disconnected vehicles carry a message, retransmit it to a new neighbor and discards that message.

\subsubsection{Vehicle density-based emergency broadcasting (VDEB)}

In [TJCWL], possible forwarders are partitioned into several rings. Ring width is included in the message. The length of waiting time is increased for each ring number. Vehicles in the outermost ring have shortest delay so they retransmit first. If retransmission fails, vehicles in the next ring retransmit. Collisions by vehicles from the same ring are resolved by 802.11 like backoff mechanism. Ring width is inversely proportional to the vehicle density (the number of neighboring vehicles detected via periodic beacon messages).

\subsubsection{Topology assisted geo-opportunistic routing (TO-GO)}

$T O-G O$ [LLG] discusses small scale routing as part of large scale one. Greedy advance is made toward the next intersection on the selected route, instead of using actual destination (not necessarily located on the same road segment) as the target destination. Forwarding targets the neighbor with advance and having the largest degree (number of its neighbors); however its location is an estimate based on beacons and may be inaccurate. Candidate receivers apply delay function that favors nodes close to the position of selected target node (an ellipse around the target node is applied to show the limits), and the one with the smallest delay will respond first offering to forward the message. For large scale routing, TO-GO applies existing recovery scheme, similar to the one originally proposed in [BMSU]. 


\subsection{Large scale routing}

Recall that large scale routing deals with forwarding decisions made at road intersections.

\subsubsection{Distance aware epidemic routing (DAER)}

Epidemic routing is a class of protocols, where a packet is forwarded to some of the neighbors. Flooding refers to forwarding by all neighbors. In [HLLLLS], a network is made up of 4000 taxis in Shanghai, with real traces. The authors propose an improvement to the epidemic routing algorithm that restricts flooding. Each receiving car will forward the packet (at most once) if it is located closer to destination than the sender. This solution is not based on the road map and movement directions. When a node needs to carry a new packet but has a full buffer, it drops the packet that has the largest number of hops and replaces it with the new one.

\subsubsection{Connectivity-aware routing (CAR)}

A position-based routing scheme called Connectivity-Aware Routing $(C A R)$ is proposed in [NG]. It targets urban areas where large scale routing is needed, and aims to mainly achieve high reliability. In this protocol, beaconing is adaptive: the fewer neighbors, the more frequent beacons. Data packets also carry beacon equivalent reports. The algorithm first performs flooding from a source $S$ to discover a destination $D$, with the delay recorded on the path, the best path is selected at the destination and reported back to the source. Routing from $S$ to $D$ then proceeds along the constructed path and therefore decisions at each road intersections (called anchors, e.g. $A, B, C$, and $E$ in Fig. 5) are predetermined. Anchor points are included in packets for changing roads. To propagate packets between anchors points, greedy forwarding is used, where current vehicle transmits the message to the neighbor closest to the next anchor point.
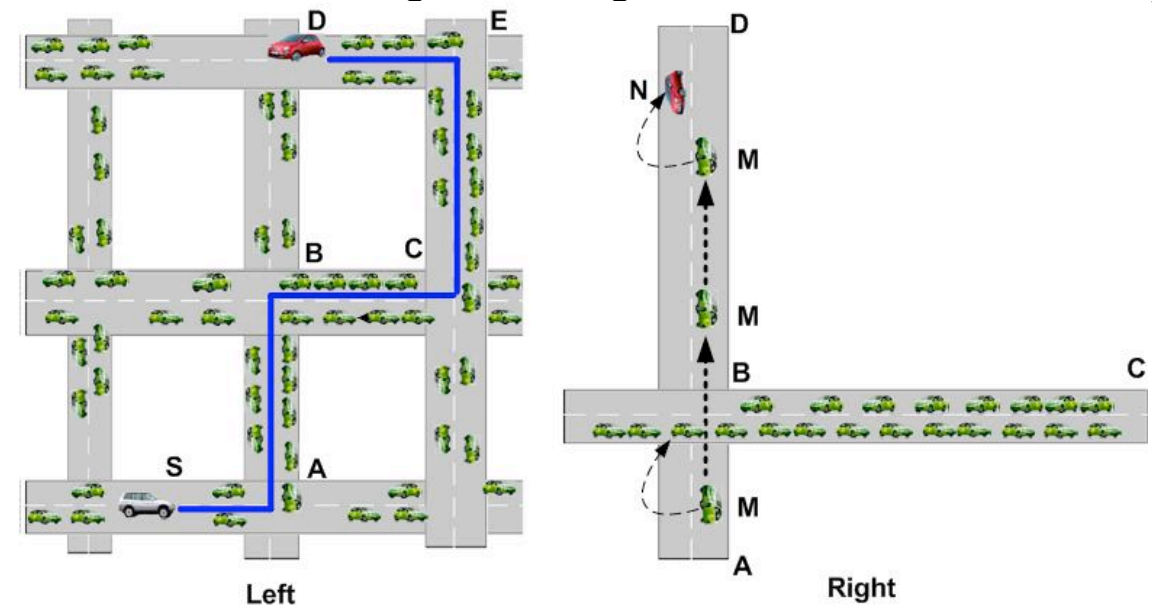

Fig. 5: $C A R$ - path construction and anchor points illustration.

If a disconnection occurs, intermediate vehicles may carry the message for a limited time until advancement to another vehicle is possible. If a vehicle carrying a message continues to drive (at anchor point) along a road which is not on predetermined path then it waits until a vehicle driving in the opposite direction (thus toward the anchor point) is able to take over the routing task. That vehicle will recursively have the same task at the anchor point. Therefore message is kept around an anchor point, by delivering to a vehicle going toward it, until a vehicle carrying the message continues driving on the selected path. In Figure 5 (left), the routing path is constructed between the source $S$ and the destination $D$ using the anchor points $A, B, C$, and $E$. Preprocessing flooding step selected this path over $A B D$ shortcut because intermittent 
disconnectivity at segment $B D$ was requiring more time to deliver a message than roads $B C D E$ where vehicles were connected at the time of flooding. Figure 5 (right) shows vehicle $M$ driving from $A$ towards $D$. $M$ transmits packet to vehicle at the anchor point (road intersection) $B$ which is driving along desired road segment $B C$. However, if none of receiving vehicles around $B$ continues driving along $B C$ then $M$ mules the message until it meets a vehicle ( $N$ in this example) driving back toward $B . N$ has recursively the same task at intersection $B$.

When destination moves, one hop route extension is possible. New location discovery is initiated if message is not delivered in certain time. It starts at an intermediate node, with flooding of half hop count of the original path. CAR algorithm may cause high message overhead because of flips around anchor points, and it has no flexibility to use opportunistic vehicles providing immediate advance along different roads where disconnection can be currently short.

\subsubsection{Delay-bounded routing in VANETs (D-greedy)}

The protocol in [ST] targets urban areas and aims to support routing to propagate a message, within a delay bound, along multiple hops toward a road-side-unit (RSU). Each vehicle is assumed to have a GPS device, a street map, a neighbor list obtained by periodic beacons, and a shortest path table. The delay-bounded greedy forwarding (D-greedy) algorithm assumes that the best path is the shortest path and each edge on the shortest path to RSU is allocated a delay budget that is proportional to its length. The protocol uses two forwarding strategies. Data muling is used if the allocated delay is sufficient while driving on a road segment; otherwise, multi-hop forwarding is used to speed up the transmission until the delay is acceptable. Multihop forwarding is also used if a vehicle carrying a message moves away from an RSU. Each edge is annotated with a cost metric that represents the number of transmissions and a delay metric that represents the time required to forward a message.

D-minCost algorithm, used in this protocol, leverages the knowledge of global traffic statistics, average speeds and vehicle density on all edges. It computes information related to delay-constrained and least-cost paths from a vehicle's location to all access points, and encodes this information in the message header. If a selected edge has no available vehicles to take over the message, the path is recomputed to find an alternative edge. Acknowledgments are used to inform the sender that the message was received so that the sender can empty the buffer used in the store-carry-forward scheme.

This algorithm has several drawbacks. First, a vehicle at an intersection carrying a message needs to find other message-carrying vehicles in a timely manner. However, it may not be able to easily find another carrier especially if the traffic is not dense. Thus, re-computation does not solve the problem efficiently. Anchor point mechanism [NG], which is explained earlier, appears to be a better option. Next, there is no mechanism for recovery when a message cannot progress toward an RSU at desired speed. After a few hops, data muling could be the only option when a vehicle desires to speed up message forwarding, as all neighboring vehicles could move away from the RSU. Vehicles driving in opposite directions are not utilized. The solution, therefore, remains incomplete.

\subsubsection{Vehicle-assisted data delivery in VANETs (VADD)}

VADD [ZC] calculates probabilities for a car arriving at intersection $i$ to pass a message to a car at road $j$ immediately, based on traffic distribution from a central delay matrix for all roads. A car arriving at an intersection selects, among neighbors on roads reducing delay, the one with the lowest expected delay. The best option could be to carry the message further, which could even drive it away. 
Although $V A D D$ aims to overcome the intermittent connectivity problem without adding new hardware, it doesn't consider that a message could be driven away from a destination and therefore lacks providing the optimal path. For example, if a forwarding vehicle is discovered at an intersection and this vehicle changes its direction, the transmitted message will not be delivered to the destination. Another problem is network overhead caused by depending on roads with dense traffic. The anchor point method proposed in [NG] could be a better option since the message will stay at an intersection until a forwarder is found.

\subsubsection{Trajectory-based data forwarding for light-traffic in VANETS (TBD)}

Large scale routing algorithm TBD [JGGSD] improves the VADD protocol. The protocol focuses on data delivery from a vehicle to an access point (RSU) with minimum time delay. A road map, vehicle arrival rates and speed for each road are available to vehicles in the area. Vehicles also know their own planned movement trajectory but do not know plans for other vehicles. A vehicle at an intersection needs to determine whether to forward a message to another vehicle or carry it. Each vehicle calculates the EDD (expected delivery delay) to the closest RSU, and adds it to its periodic beacon to inform neighbors. The EDD is calculated assuming constant vehicle speed, few wireless hops initially from RSU, and carrying messages until the next intersection. The algorithm sorts roads at intermediate intersections by geographically shortest paths, to establish forwarding priority. Thus, a message is forwarded to a vehicle with minimum $E D D$. Instead of returning the message to anchor points as in [NG], the advance in [ZC, JGGSD] is attempted at the next intersection.

The protocol uses simple broadcasting to exchange the $E D D$ with neighbors causing a broadcast storm which increases communication delay and network overhead. Another problem is relying on information such as speed, trajectory and direction to predict the time delay, and this information is not stable.

An alternative to EDD calculation is to route an actual beacon message between RSUs [DWX] by using one of small scale routing algorithms. These delays are then uploaded in a central matrix, and shortest paths are derived from this matrix. To increase reliability in case of sparse traffic, routing is proposed over two best paths from RSUs [DWX].

\subsubsection{A static-node assisted adaptive routing protocol in VANETs (SADV)}

$S A D V$ is a large scale routing protocol to overcome the intermittent connectivity problem by using static nodes (RSUs) at intersections [DWX]. This protocol makes use of the link delay update and the multiple-path forwarding to reduce the time delay. In the link delay update, adjacent nodes periodically compute the link delay between each other to adaptively determine the best path with minimum time delay. The calculated time delays are based on real time traffic density. In multiple-path forwarding, a static node at an intersection forwards the message to adjacent static nodes on different paths. This increases the chance of hitting the optimal path. To minimize network overhead, multiple paths are used only at an intersection and each RSU remembers its transmitted message for a time period and discards duplicated messages. SADV protocol requires buffer management to eliminate unneeded messages, and installing new hardware (RSUs).

\subsubsection{Location- and delay-aware cross-layer communication (LD-CROP)}

LD-CROP algorithm [JE] relies on periodic beacons, initiated by a RSU, with packets related to traffic characteristics. Beacons contain street/direction path and path quality (statistics 
over sliding time window). They are forwarded by the farthest receiver after defer time delay (cross-layer design) with updated path statistics and an appended own location (if located at an intersection). Source routing towards RSU is applied over smaller delay paths. A source vehicle decides the series of streets. Dynamic data forwarding decisions allow paths to be changed at other intersections. Neighbors bundle multiple packets, with higher priority to forward. Farther neighbors apply smaller waiting time to forward. The algorithm remains incomplete at intersections if there is no vehicle providing advance in the desired direction. Vehicles from opposite direction are not used. Simulation is carried on a $3 \times 3$ road map due to scalability issue.

\subsubsection{Geographical opportunistic routing (GeoOpps)}

In the routing GeOpps scheme in [LM], the destination is a fixed geographic location. Cars are assumed to exchange their planned paths with neighbors. Then, the nearest point (NP) to the destination along the trajectory is found. The time to drive to the NP plus the time for a car to drive from the NP to the destination $D$ is measured for all neighbors. A message is forwarded to a neighbor having the smallest measure. This favors fast-driving cars, not necessarily cars whose NP is closest possible to the destination. The segment from NP to the destination is unpredictable, and different NPs may behave quite differently, including closed or empty roads. The algorithm may have loops because the metric used in non-monotone [CRR], and therefore there is no guaranteed delivery even with well populated roads. The routing algorithm in [LM] was applied in $[\mathrm{LCM}]$ to preserve content in a persistence area before a vehicle arrives to an opportunistic area. This is achieved by routing message replica periodically to its home zone. However, these message replicas do not reach all the nodes in the persistence area, and there is no automatic transfer of content when holders leave the area.

\subsubsection{Road-based vehicular traffic routing (RBVT)}

In [NRWB], proactive routing is based on Depth First Search (DFS) traversal to compute the shortest path along a road network. Receivers compete for relaying with a delay function that depends on forwarding progress, received power, and transmission area. Constructed route can be maintained by extending (adding) or deleting intersections when source or destination is mobile. Temporary disconnection and store-and-forward approach are not discussed in [NRWB]. Route discovery is based on 'one-way' broadcasting which may 'jump' over cars on other roads. Route can be maintained by extending or cutting intersections for mobile source or destination.

\subsubsection{Improved greedy traffic-aware routing protocol (GyTAR)}

Another example is in [JSRG] where a decentralized estimation of traffic density is discussed. A road network is divided into grids, and the average numbers of cars per grid and the standard deviation are calculated. The next intersection is selected based on the weighted sum of its score distance (defined as the reduction ratio of distance to $D$ ) and the traffic density. Note that 'score distance' has lower impact when a sender is far from $D$. Greedy forwarding as in [SFLYOF] is applied between intersections. Positions of neighbors are predicted. Carry and forward is used as a recovery mode, if no vehicle closer to the next intersection is identified.

\subsubsection{Access-overlay routing by two phase routing protocol (TOPO)}

The TOPO algorithm consists of access routing phase and overlay routing phase [WXC]. $T O P O$ constructs an overlay network of roads with fast moving vehicles (highways). Other roads are considered as access network for the overlay and are connected to the overlay via access 
points. Routing proceeds from the source to the access point (AA), from access point to the overlay (AO), along overlay (OO), from overlay to access point (OA) and from access point to the destination (AA). Therefore access-overlay (AO) nodes are part of path as temporary destinations. Message is bounced around AO and OA intersections until some node is able to get it to desired road.

\subsection{Summary}

The table below shows a summary of our classification. The abbreviations used in the table represent the taxonomy explained earlier in this paper. Here, we review our taxonomy and show the corresponding abbreviations.

1- Problem statement: Geocasting $(G)$ or Routing $(R)$. A routing task can be decomposed into Small Scale routing $(S S)$, Large Scale routing $(L S)$ and Finding a Destination $(F D)$ steps.

2- Transmission dimensions: One Dimension road (1D), or Two Dimensions (2D).

3- Neighbor knowledge: Neighbor Positional (NP) knowledge is required, or No Neighbor knowledge $(\mathrm{NN})$.

4- Acknowledgment: No Acknowledgments (NA), Beacon Acknowledgment (BA), Passive Acknowledgment (PA), or Reception Estimation (RE).

5- Starting forwarder selection: Always Activate (AA), Immediate Activate (IA), Lack of Acknowledgment (LA), or Neighbor Elimination (NE).

6- Compete to retransmit: Not competing (NC), Time Delay (TD) based on DSRC (TD-D) or RTS CTS (TD-R), or by Black Burst $(B B)$.

7- Connectivity: Intermittent Connectivity (IC) is addressed, or vehicles are Assumed Connected $(A C)$ all the time.

8- Urgency: Time Critical (TC) for emergency messages, Reliability Oriented (RO) for nonurgent messages.

9- Message content: Full Message (FM) is immediately sent, or Forwarder ID is Attached (FA) with the message.

10- Some protocols have incomplete description regarding some categories in our taxonomy, which is denoted by $\mathrm{X}$ in the table.

Table 2: Taxonomy of VANET data communication protocols

\begin{tabular}{|c|c|c|c|c|c|c|c|c|c|c|c|}
\hline 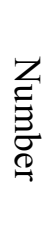 & $\begin{array}{l}7 \\
\overrightarrow{0} \\
0 \\
0 \\
0 \\
0\end{array}$ & 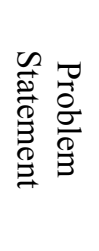 & 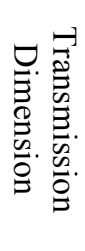 & 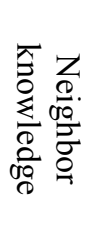 & 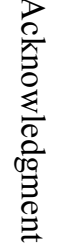 & 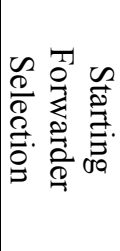 & 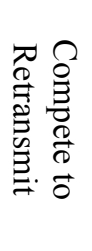 & 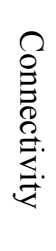 & 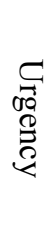 & $\begin{array}{l}3 \\
0 \\
0 \\
0 \\
00 \\
00 \\
0 \\
0 \\
0 \\
\stackrel{0}{0} \\
\stackrel{0}{0}\end{array}$ & $\begin{array}{l}\overrightarrow{0} \\
0 \\
0 \\
0 \\
0 \\
0 \\
0\end{array}$ \\
\hline 1 & $\begin{array}{l}\text { Probabilistic, } \\
\text { naïve } \\
\text { flooding }\end{array}$ & G & $2 \mathrm{D}$ & NN & NA & AA & $\mathrm{NC}$ & IC & RO & FM & $\begin{array}{l}{[\mathrm{FM},} \\
\text { BTD] }\end{array}$ \\
\hline 2 & Middle & G & 1D & $\mathrm{NN}$ & NA & IA & TD-D & $\mathrm{AC}$ & RO & FA & [LLZ] \\
\hline 3 & $A c k-P B S M$ & G & $2 \mathrm{D}$ & NP & BA & LA,NE & TD-D & IC & RO & FM & [RRS] \\
\hline 4 & Persistence & $\mathrm{G}$ & 1D & $\mathrm{NN}$ & PA & IA & TD-D & $\mathrm{AC}$ & RO & FM & [WTPMBS] \\
\hline 5 & $M H V B$ & G & 1D & $\mathrm{NN}$ & PA & $\mathrm{NE}$ & TD-D & $\mathrm{AC}$ & $\mathrm{TC}$ & FM & [OLL] \\
\hline 6 & $E M D O R$ & $\mathrm{G}$ & $1 \mathrm{D}$ & $\mathrm{NN}$ & $\mathrm{BA}$ & IA, LA & TD-D & $\mathrm{AC}$ & $\mathrm{TC}$ & FA & {$[\mathrm{SYK}]$} \\
\hline 7 & $E M D V$ & $\mathrm{G}$ & $2 \mathrm{D}$ & NP & $\mathrm{RE}$ & IA & TD-D & $\mathrm{AC}$ & $\mathrm{TC}$ & FA & [TMSH] \\
\hline 8 & $\operatorname{ReC}$ & $\mathrm{G}$ & $2 \mathrm{D}$ & NP & $\mathrm{BA}$ & LA,NE & TD-D & IC & $\mathrm{TC}$ & FM & {$[\mathrm{LYS}]$} \\
\hline
\end{tabular}




\begin{tabular}{|c|c|c|c|c|c|c|c|c|c|c|c|}
\hline 9 & OPERA & R-SS & $1 \mathrm{D}$ & NP & BA & $\mathrm{NE}$ & $\mathrm{X}$ & IC & $\mathrm{TC}$ & FM & [AOS] \\
\hline 10 & $B P A B$ & R-SS & 1D & NN & PA & IA & BB, TD-R & $\mathrm{AC} / \mathrm{IC}$ & $\mathrm{TC}$ & FA & [SWSG] \\
\hline 11 & $T R A D E / D D T$ & R-SS & 1D & NP/NN & NA & IA & TD-D & $\mathrm{AC}$ & $\mathrm{RO}$ & FA & [SFLYOF] \\
\hline 12 & $C B R F$ & R-SS & $1 \mathrm{D}$ & $\mathrm{NN}$ & $\mathrm{BA}$ & IA & TD-R & $\mathrm{AC}$ & TC & FA & {$[\mathrm{WXC}]$} \\
\hline 13 & $D P P$ & R-SS & 1D & NP & $\mathrm{BA}$ & IA & TD-D & IC & $\mathrm{TC}$ & FM & {$[\mathrm{LA}]$} \\
\hline 14 & $D V-C A S T$ & R-SS & $1 \mathrm{D}$ & NP & PA & $\mathrm{I}$ & TD-D & $\mathrm{AC}$ & $\mathrm{RO}$ & FM & [TWB] \\
\hline 15 & $V D E B$ & R-SS & 1D & NP & BA & IA & TD-D & $\mathrm{AC}$ & $\mathrm{TC}$ & FA & [TJCWL] \\
\hline 16 & TOGO & R-SS & $1 \mathrm{D}$ & NP & BA & IA & TD-D & IC & RO & FM & [LLG] \\
\hline 17 & DAER & R-LS & $2 \mathrm{D}$ & NP & BA & IA & TD-D & $\mathrm{AC}$ & $\mathrm{RO}$ & FM & [HLLLLS] \\
\hline 18 & $C A R$ & R-FD, LS & $2 \mathrm{D}$ & NP & BA & IA & TD-R & IC & $\mathrm{RO}$ & FM & {$[\mathrm{NG}]$} \\
\hline 19 & D-greedy & R-LS & $2 \mathrm{D}$ & NP & BA & IA & TD-D & IC & TC & FM & {$[\mathrm{ST}]$} \\
\hline 20 & $V A D D$ & R-LS & $2 \mathrm{D}$ & NP & BA & IA & TD-D & IC & $\mathrm{TC}$ & FM & {$[\mathrm{ZC}]$} \\
\hline 21 & $T B D$ & R-LS & $2 \mathrm{D}$ & NP & BA & IA & TD-R & IC & $\mathrm{TC}$ & FM & [JE] \\
\hline 22 & $S A D V$ & R-LS & $2 \mathrm{D}$ & NP & $\mathrm{BA}$ & IA & TD-D & IC & $\mathrm{TC}$ & FM & {$[\mathrm{DWX}]$} \\
\hline 23 & $L D-C R O P$ & R-LS & $2 \mathrm{D}$ & NP & BA & IA & TD-R & IC & $\mathrm{RO}$ & FM & [JGGSD] \\
\hline 24 & GeOpps & R-LS & $2 \mathrm{D}$ & NP & $\mathrm{BA}$ & IA & TD-D & IC & $\mathrm{TC}$ & FA & [LM] \\
\hline 25 & $R B V T$ & R-FD, LS & $2 \mathrm{D}$ & NP & $\mathrm{BA}$ & IA & TD-D & $\mathrm{AC}$ & $\mathrm{RO}$ & FA & [NRWB] \\
\hline 26 & GyTAR & R-LS & $2 \mathrm{D}$ & NP & $\mathrm{BA}$ & IA & TD-D & IC & RO & FA & [JSRG] \\
\hline 27 & TOPO & R-LS & $2 \mathrm{D}$ & NP & $\mathrm{BA}$ & IA & TD-R & $\mathrm{IC}$ & RO & FA & [WXC] \\
\hline
\end{tabular}

\subsection{Conclusion and future work}

We have reviewed VANET applications and provided novel classification of VANET data communication protocols. Our taxonomy includes the problem statement of each protocol and other characteristics such as road dimension, neighbor knowledge, acknowledgment, start of forwarding, competition to retransmit, vehicle connectivity, urgency, and message contents. We extensively surveyed the most relevant data communication protocols proposed to address different VANET applications in different scenarios. We have explained, characterized and compared these protocols to highlight their drawbacks while outlining possible solutions.

The presented taxonomy helps researcher understand the details of VANET data communication protocol. It also helps to understand various limitations of certain protocols, and the need to generalize these protocols and apply them in various VANET scenarios. One of our contributions is to properly classify protocols according to the actual problem that they solve. It was observed that there is no common vocabulary for the problem statements, so it is not easy to find proper competitors for various protocols. For example, [SFLYOF] claims to describe 'broadcast' algorithms. However, since there is no attempt to provide reliability between two transmitters, and the main goal is to advance message propagation in certain direction, it is more appropriate to be classified as small scale routing algorithm. The incompleteness of certain protocols can be also observed using the taxonomy. For example, OPERA [AOS] is lacking MAC layer (and has ' $\mathrm{X}$ ' under 'compete to retransmit').

Our taxonomy hints to limitations of certain protocols. Protocols addressing 1D scenario are limited compared to 2D ones. Protocols such as in [OLL] [WTPMBS] [SYK] [SWSG] [SFLYOF] [WXC] [LA] [TWB] [TJCWL] are 1D and remain incomplete when applied to 2D scenario. Protocols addressing 2D case are more general, and are also applicable when the actual scenario is $1 \mathrm{D}$. Further, protocols based on beacons (BA), such as ackPBSM [RRS] and D-FPAV [TMSH], have communication overhead which needs to be considered and could potentially limit the performance. In order to reduce beacons overhead, other protocols such as Persistence-based protocols [WTPMBS] and MHVB [OLL] do not depend on beacons since they do not require neighbor knowledge or beacon acknowledgments. Instead they use passive acknowledgments $(P A)$ or no acknowledgments (NA). However, the reliability of these protocols may decrease [RRS].

The next limitation of many protocols is long time delay. It is caused when a node applies long waiting time before it retransmits which will delay messages from reaching the destination. 
In $M H V B$ [OLL], the waiting time is increased in order to check whether the vehicle is in congestion or not. In $B P A B$ [SWSG], a delay is caused by waiting the forwarder to determine the relay node. The time delay also increases by vehicles driving away from the desired path [ZC] or by keeping the message around an intersection hoping to find a vehicle driving toward the destination [JE].

Protocols assuming always connected scenarios (AC) are limited compared to more general protocols handling intermittent connectivity (IC). When the forwarding area is empty of vehicles, message will not be delivered to the destination. This problem appears in [OLL] [WTPMBS] [SYK] [TMSH] since they do not provide any mechanism to keep the message alive at disconnection. Also, other protocols do not consider that a vehicle carrying the message may drive far away from the desired path as VADD [ZC] or do not utilize vehicles in the opposite direction as D-greedy [ST]. Other protocols as in [RRS] [AOS] [NG] use the store-carry-forward scheme to overcome the intermittent connectivity problem.

Immediate activation $(I A)$ and always activation (AA) do not resolve properly collision issues. In [WTPMBS] and [SYK], all nodes within the same slot may become active once they receive a message and retransmits together. In ackPBSM [RRS], collision is prevented by using lack of acknowledgment $(L A)$ and neighbor elimination $(N E)$ which allows a node to be active if it has a neighbor who has not yet received the message.

The next common problem in many protocols is the possibility of collision due to selecting same time delay (TD-D). Nodes may apply the same waiting time before they retransmit which causes collision as in [WTPMBS]. MAC protocols based on 802.11 standards resolve this by adding a random backoff time to each node before transmission begins, as in [TJCWL].

For designing effective protocols, the taxonomy highlights some fundamental concepts. First, protocols should aim at general scenarios, addressing 2D and intermittent connectivity. Next, protocols should minimize communication overheads and time delays. Although communication overheads can be reduced by reducing the dependency on beacons through using passive acknowledgments (PA) or reception estimation (RE), the experiments such as [RRS] show that explicit beacon acknowledgments provide very high reliability at small increase in communications. Time delay can be reduced by enabling vehicles to explore multiple paths in order to determine the optimal one or by utilizing intersections as in the anchor-points scheme. This again increases communication overheads.

New protocols can be designed by replacing one ingredient in a category by another one. In most cases, TD-D, TD-R and BB methods for competing to retransmit are interchangeable, without affecting other parts of protocol. Next, new protocols can be designed by taking ingredients from the corresponding basic protocols. For instance, a small scale routing algorithm (R-SS) can be combined with a large scale one (R-LS) to arrive at new routing algorithm.

\section{References}

[AOS] M. Abuelela, S. Olariu, and I. Stojmenovic, "OPERA: Opportunistic Packet Relaying in Disconnected Vehicular Ad Hoc Networks," 5th IEEE International Conference on Mobile Ad Hoc and Sensor Systems MASS Atlanta, USA, 2008.

[BM] J. Bernsen and D. Manivannan, "Unicast Routing Protocols for Vehicular Ad hoc Networks: A critical Comparison and Classification," Pervasive and Mobile Computing, vol. 5, pp. 1-18, 2009.

[BMSU] P. Bose, P. Morin, I. Stojmenovic and J. Urrutia, "Routing with Guaranteed Delivery in Ad Hoc Wireless Networks," ACM Wireless Networks, vol. 7, no. 6, pp. 609-16, Nov. 2001.

[BTD] S. Biswas, R. Tatchikou, and F. Dion, "Vehicle-to-Vehicle Wireless Communication Protocols for Enhancing Highway Traffic Safety," IEEE Commun. Mag., vol. 44, pp. 74-82, Jan 2006. 
[BF] I. Broustis and M. Faloutsos, "Routing in Vehicular Networks: Feasibility, Modeling, and Security," International Journal of Vehicular Technology, pp. 1-8, 2008.

[CGM] M. Caliskan, D. Graupner, and M. Mauve, "Decentralized Discovery of Free Parking Places," 3rd ACM Int. Workshop VANET, Los Angeles, CA, pp. 30-39, Sep 2006.

[CNS] A. Casteigts, A. Nayak, and I. Stojmenovic, "Communication Protocols for Vehicular Ad hoc Networks," Wireless Communications and Mobile Computing, 11 (5), May 2011, 567-582.

[CRR] V. Cabrera, F. J. Ros, and P. M. Ruiz, "Simulation-Based Study of Common Issues in VANET Routing Protocols," IEEE 69th Vehicular Technology Conference, Barcelona: IEEE, 2009.

[DWX] Y. Ding, C. Wang, and L. Xiao, "A Static-Node Assisted Adaptive Routing Protocol in Vehicular Networks," ACM VANET, New York, pp. 59-68, 2007.

[DDB] M. Durresi, A. Durresi, and L. Barolli, "Emergency Broadcast Protocol for Inter-vehicle Communications," 11th ICPADS, Fukuoka, Japan, vol. 2, pp. 402-406, Jul 2005.

[FM] R. Fracchia and M. Meo, "Analysis and Design of Warning Delivery Service in Inter-vehicular Networks," IEEE Transactions on Mobile Computing, vol. 8, pp. 832-845, 2008.

[HLLLS] H.-Y. Huang, P.-E. Luo, M. Li, D. Li, X. Li, W. Shu, and M.-Y. Wu, "Performance Evaluation of SUVnet with Real-Time Traffic Data," IEEE Transactions on Vehicular Technology, vol. 56, pp. 3381-3396, 2007.

[JE] B. Jarupan and E. Ekici, "Location- And Delay-Aware Cross-Layer Communication in V2I Multihop Vehicular Networks," IEEE Communications Magazine, pp. 112-118, Nov 2009.

[JGGSD] J. Jeong, S. Guo, Y. Gu, T. He, and D. H. C. Du, "TBD: Trajectory-Based Data Forwarding For LightTraffic Vehicular Ad-Hoc Networks," 29th IEEE International Conference on Distributed Computing Systems, pp. $231-238$, Nov 2009.

[JSRG] M. Jerbi, S.-M. Senouci, T. Rasheed, and Y. Ghamri-Doudane, "Towards Efficient Geographic Routing in Urban Vehicular Networks," IEEE Transactions on Vehicular Technology, vol. 58, pp. 5048 - 5059, 2009.

[LA] T. D. C. Little and A. Agarwal, "An Information Propagation Scheme for Vanets," IEEE Intelligent Transportation Systems, 2005, pp. 155-160.

[LCM] I. Leontiadis, P. Costa, and C. Mascolo, "Persistent Content-Based Information Dissemination in Hybrid Vehicular Networks " IEEE Int. Conf. Pervasive Computing and Communications PerCom, 2009.

[LLG] K.C. Lee, U. Lee, and M. Gerla, "Geo-opportunistic routing for vehicular networks", IEEE Communications Magazine, May 2010, 164-170.

[LLZ] M. Li, W. Lou, and K. Zeng, "OppCast: Opportunistic Broadcast of Warning Messages in VANETs with Unreliable Links," 6th IEEE International Conference on Mobile Adhoc and Sensor Systems MASS, 2009.

[LM] I. Leontiadis and C. Mascolo, "GeOpps: Geographical Opportunistic Routing for Vehicular Networks," Workshop on Autonomic and Opportunistic Communications, IEEE WoWMoM Helsinki, 2007.

[LYS] J. Liu, Z. Yang, I. Stojmenovic, "Receiver consensus: Rapid and reliable broadcasting for warning delivery," 32nd IEEE International Conference on Distributed Computing Systems ICDCS, Macau, June 18-21, 2012.

[NG] V. Naumov and T. R. Gross, "Connectivity-Aware Routing (CAR) in Vehicular Ad Hoc Networks," 26th IEEE International Conference on Computer Communications INFOCOM Anchorage, AK, 2007.

[NRWB] J. Nzouonta, N. Rajgure, G. Wang, and C. Borcea, "VANET Routing on City Roads Using Real-Time Vehicular Traffic Information," IEEE Transactions on Vehicular Technology, vol. 58, pp. 3609 - 3626, 2009.

[NSI] T. Nadeem, P. Shankar, and L. Iftode, "A Comparative Study of Data Dissemination Models for Vanets," 3rd Annu. Int. Conf. MOBIQUITOUS, San Jose, CA, pp. 1-10, Jul 2006.

[OLL] T. Osafune, L. Lin, and M. Lenardi, "Multi-Hop Vehicular Broadcast (MHVB)," 6th International conference on ITS Telecommunications, Chengdu, 757-760, 2006.

[RRS] F. Ros, P. Ruiz, and I. Stojmenovic, "Acknowledgment-based broadcast protocol for reliable and efficient data dissemination in vehicular ad-hoc networks," IEEE Transactions on Mobile Computing, Volume 11, No. 1, 2012, 33-46; IEEE Vehicular Technology Conference VTC Barcelona, 2009.

[RRSS] F. J. Ros, P. M. Ruiz, J. A. Sanchez, and I. Stojmenovic, "Mobile Ad Hoc Routing in The Context of Vehicular Networks, in Handbook of Vehicular Networks," S. Olariu and M. Weigle, Eds.: Chapman \& Hall/CRC/ T\&F, Chapter 9, 1-48, 2009.

[SFLYOF] M.-T. Sun, W.-c. Feng, T.-H. Lai, K. Yamada, H. Okada, and K. Fujimura, "GPS-based Message Broadcast for Adaptive Inter-Vehicle Communications," IEEE VTC, 52nd Boston, MA, USA, 2000.

[SSZ] I. Stojmenovic, M. Seddigh, and J. Zunic, "Dominating sets and Neighbor Elimination-Based Broadcasting Algorithms in Wireless Networks," IEEE Transactions on Parallel and Distributed Systems, vol. 13, pp. 14-25, 2002.

[ST] A. Skordylis and N. Trigoni, "Delay-Bounded Routing in Vehicular Ad-Hoc Networks," ACM MobiHoc, 
Hong Kong, pp. 341-350, May 2008.

[SWSG] J. Sahoo, E. H. K. Wu, P. K. Sahu, and M. Gerla, "BPAB: Binary Partition Assisted Emergency Broadcast Protocol For Vehicular ad hoc Networks," 18th Int. Conf. Computer Communications and Networks ICCN, San Francisco, CA 2009.

[SYK] D. Shin, H. Yoo, and D. Kim, "Emergency Message Dissemination with Ack-Overhearing Based Retransmission," First International Conference on Ubiquitous and Future Networks Hong Kong, 2009.

[TJCWL] Y.T. Tseng, R.H. Jan, C. Chen, C.F, Wang, H.H. Li, "A Vehicle-density-based Forwarding Scheme for Emergency Message Broadcasts in VANETs," IEEE 7th International Conference on Mobile Adhoc and Sensor Systems (MASS), San Francisco, USA, Nov. 2010.

[TMSH] M. Torrent-Moreno, J. Mittag, P. Santi, and H. Hartenstein, "Vehicle-to-Vehicle Communication: Fair Transmit Power Control for Safety-Critical Information," IEEE Trans. Vehicular Technology, vol. 58, pp. 3684-3703, Sept 2009.

[TWB] O. Tonguz, N. Wisitpongphan, F. Bai, "DV-CAST: A distributed vehicular broadcast protocol for vehicular ad hoc networks," IEEE Wireless Communications Magazine, pp. 47-56, April 2010.

[WER] L. Wischhof, A. Ebner, and H. Rohling, "Information Dissemination in Self-Organizing Inter-vehicle Networks," IEEE Trans. Intell. Transp. Syst., vol. 6, pp. 90-101, Mar 2005.

[WTM] T. Willke, P. Tientrakool, and N. Maxemchuk, "A Survey of Inter-Vehicle Communication Protocols and Their Applications," IEEE Communications Surveys \& Tutorials, vol. 11, pp. 3-20, 2009.

[WTPMBS] N. Wisitpongphan, O. K. Tonguz, J. S. Parikh, P. Mudalige, F. Bai, and V. Sadekar, "Broadcast Storm Mitigation Techniques in Vehicuar Ad Hoc Wireless Networks," IEEE Wireless Communications Magazine, pp. 84-94, Dec 2007.

[WXC] W. Wang, F. Xie, and M. Chatterjee, "Small Scale and Large Scale Routing in Vehicular Ad Hoc Networks," IEEE Transactions on Vehicular Technology, vol. 58, pp. 5200-5213, 2009.

[YMF] S. Yousefi, M. S. Mousavi, and M. Fathy, "Vehicular Ad Hoc Networks (VANETs): Challenges and Perspectives," IEEE International Conference on ITS Telecommunications, Chengdu, 2006, pp. 761 - 766.

[ZC] J. Zhao and G. Cao, "VADD: Vehicle-Assisted Data Delivery in Vehicular Ad Hoc Networks," IEEE Transactions on Vehicular Technology, vol. 57, pp. 1-12, May 2008. 